An Optical Time Delay Estimate for the Double Gravitational Lens System B1600+434

This article has been downloaded from IOPscience. Please scroll down to see the full text article.

2000 ApJ 544117

(http://iopscience.iop.org/0004-637X/544/1/117)

The Table of Contents and more related content is available

Download details:

IP Address: 139.165.20.112

The article was downloaded on 02/04/2010 at 15:51

Please note that terms and conditions apply. 
The AstrophysicAL JournaL, 544:117-122, 2000 November 20

(c) 2000. The American Astronomical Society. All rights reserved. Printed in U.S.A.

\title{
AN OPTICAL TIME DELAY ESTIMATE FOR THE DOUBLE GRAVITATIONAL LENS SYSTEM $\mathrm{B} 1600+434^{1}$
}

\author{
I. BURUD \\ Institut d'Astrophysique et de Géophysique de Liège, Avenue de Cointe 5, B-4000 Liège, Belgium \\ J. HJORTH \\ Astronomical Observatory, University of Copenhagen, Juliane Maries Vej 30, DK-2100 Copenhagen Ø, Denmark
}

A. O. JAUNSEN

Institute of Theoretical Astrophysics, University of Oslo, Pb. 1029 Blindern, N0315 Oslo, Norway

M. I. ANDERSEN AND H. KORHONEN

Division of Astronomy, University of Oulu, PO Box 3000, FIN-90014 Oulun Yliopisto, Finland

J. W. CLASEN

Nordic Optical Telescope, Apartado 474, E-38700 Santa Cruz de La Palma, Canary Islands, Spain

J. PELT

Tartu Astrophysical Observatory, Tõravere, 61602, Estonia

F. P. PIJPERS

Theoretical Astrophysics Center, University of Aarhus, DK-8000 Århus C, Denmark

P. MAGaIN

Institut d'Astrophysique et de Géophysique de Liège, Avenue de Cointe 5, B-4000 Liège, Belgium

AND

R. ØSTENSEN

Department of Physics, University of Tromsø, Tromsø, Norway

Received 2000 June 8; accepted 2000 July 13

\begin{abstract}
We present optical I-band light curves of the gravitationally lensed double QSO B1600+434 from observations obtained at the Nordic Optical Telescope (NOT) between 1998 April and 1999 November. The photometry has been performed by simultaneous deconvolution of all the data frames, involving a numerical lens galaxy model. Four methods have been applied to determine the time delay between the two QSO components, giving a mean estimate of $\Delta t=51 \pm 4$ days $(95 \%$ confidence level). This is the fourth optical time delay ever measured. Adopting a $\Omega=0 . \overline{3}, \Lambda=0$ universe and using the mass model of Maller et al., this time delay estimate yields a Hubble parameter of $H_{0}=52_{-8}^{+14} \mathrm{~km} \mathrm{~s}^{-1} \mathrm{Mpc}^{-1}(95 \%$ confidence level), where the errors include time delay as well as model uncertainties. There are timedependent offsets between the two (appropriately shifted) light curves that indicate the presence of external variations due to microlensing.

Subject headings: cosmology: observations - distance scale - galaxies: spiral gravitational lensing — quasars: individual $(\mathrm{B} 1600+434)$

On-line material: color figures
\end{abstract}

\section{INTRODUCTION}

Intensive observational studies of gravitationally lensed QSOs have been conducted in the last few years with the aim of determining cosmological parameters, e.g., the Hubble constant, $H_{0}$, and to study the dark matter distribution in lens galaxies. In particular, there has been a significant effort to measure time delays between lensed QSO components to derive $H_{0}$ with the method described by Refsdal (1964). Following a more than decade-long monitoring of the double QSO $0957+561$ by Schild and coworkers (Schild 1990; Schild \& Thomson 1995), Schild \& Thomson (1997) and Kundic et al. (1997) succeeded in pinning down its time delay in 1997. The same year saw an even more impressive accomplishment of Schechter et al.

\footnotetext{
${ }^{1}$ Based on observations made with the Nordic Optical Telescope, operated on the island of La Palma jointly by Denmark, Finland, Iceland, Norway, and Sweden, in the Spanish Observatorio del Roque de los Muchachos of the Instituto de Astrofisica de Canarias.
}

(1997) in determining multiple time delays in the "triple QSO" PG $1115+080$ from a peak-to-peak variation of barely $0.15 \mathrm{mag}$. Following this demonstration of the feasibility of measuring time delays of multiply imaged QSOs, an ongoing photometric monitoring was initiated at the Nordic Optical Telescope (NOT) in 1998 April. The program involves measuring time delays between lensed QSO components in as many systems as possible. Our main target during the first year of monitoring was the doubly imaged radio source QSO B1600+434 (Jackson et al. 1995) at redshift $z=1.59$ (Fassnacht \& Cohen 1998), which is gravitationally lensed by an edge-on late-type galaxy at $z=0.41$ (Jaunsen \& Hjorth 1997; Fassnacht \& Cohen 1998). The 1".39 angular separation between the two QSO images (labeled A and B in Fig. 1), together with the observed large flux variations in the system (Jaunsen \& Hjorth 1997), makes it well suited to time delay measurements. Given the poor knowledge of the mass distribution in dark matter halos of spiral galaxies, a time delay measurement in $\mathrm{B} 1600+434$ may not provide a firm determi- 

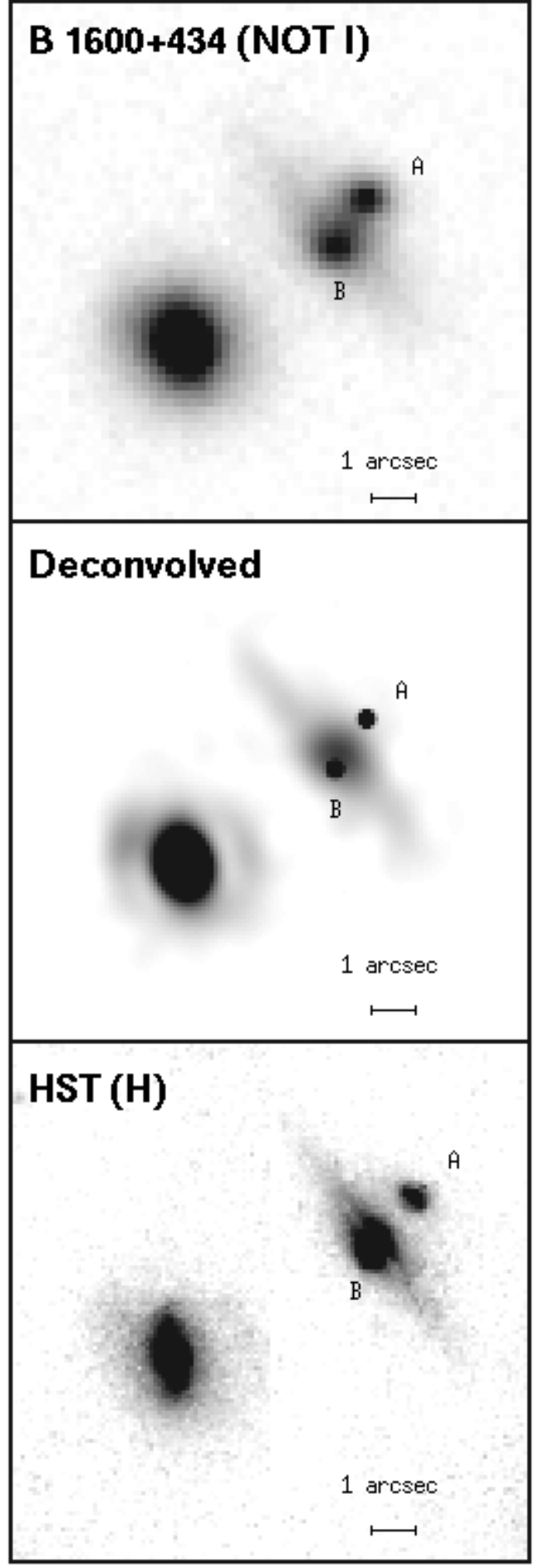

Fig. 1.-Top: Stacked I-band images of $12^{\prime \prime} \times 12^{\prime \prime}$ around B1600 +434 with a total of $\sim 3.5 \mathrm{hr}$ of exposure and a seeing of FWHM $=1$ 1.13. Middle: Image (FWHM =0"38) obtained from the simultaneous deconvolution of 33 frames. The main lensing spiral galaxy can be seen between the two QSO components A and B. A neighbor galaxy is seen to the southeast of the system. North is up and east is to the left. Bottom: HST $H$-band image.

nation of $H_{0}$. However, once $H_{0}$ has been determined from other lensed systems or using other methods, the time delay of B1600+434 can provide new constraints on the distribution of mass in the various components of spiral galaxies in general (Maller et al. 2000).
B1600+434 is very faint, with $I \sim 22$ for the faintest QSO component and $I=20.3$ for the lensing galaxy. Furthermore, the B-component is substantially obscured by the lens (see Fig. 1), and photometry of the image is nontrivial. The data have therefore been analyzed with advanced deconvolution techniques both in order to model the light distribution of the lensing galaxy and to achieve accurate photometry of the QSO component blended with the lens nucleus. The time delay we present using the deconvolution technique is the fourth one measured at optical wavelengths, and it is the first of our monitoring program. A preliminary report was presented in Hjorth et al. (2000). The results presented here include more data points and supersede this report. Independently, a time delay has been measured at radio wavelengths with the VLA during the same observing season (Koopmans et al. 2000).

\section{OBSERVATIONS AND DATA REDUCTION}

Weekly observations of B1600 +434 were carried out at the NOT from 1998 April to 1999 November, except for a short period (i.e., from 1998 December to 1999 February) when the object was below the horizon at the NOT. Three different instruments were used: the Andalucía Faint Object Spectrograph (ALFOSC), the High Resolution Adaptive Camera (HiRAC), and the standby camera StanCam, equipped with detectors yielding pixel scales of 0.188 , $00^{\prime \prime} 107$, and 0.176 , respectively. The $I$ band was chosen to minimize the effect of the extinction of the B component by the lensing galaxy. The total exposure times for each data point were adjusted according to the moon phase and typically ranged from 20 to 40 minutes, divided into three dithered exposures. The seeing usually varied from 0 ."7 to 1 ."4, with 0 ".9 being the most frequent value. A typical signal-tonoise ratio $(\mathrm{S} / \mathrm{N})$ of 100 was reached for the A component, 50 for the B component. Color terms were determined for the three detectors in order to match the light curves obtained with the three instruments.

An automated pipeline employing routines in the IRAF/ NOAO/CCDRED package has been developed in order to preprocess the CCD frames in an efficient and homogeneous way. Fringe correction and cosmic-ray removal were performed on the individual frames before combination.

\section{PHOTOMETRY}

The photometric data consist of one stacked frame per epoch. All light curves are calculated relative to three stars in the field that have calibrated $I$-band magnitudes (Jaunsen \& Hjorth 1997). Two of these stars, angularly close to the QSO, labeled S1 and S2 by Jaunsen \& Hjorth (1997), are used to construct the point-spread function (PSF).

All the combined frames, one per epoch, are deconvolved with the MCS deconvolution algorithm (Magain, Courbin, $\&$ Sohy 1998). With this technique, many frames of a single object can be simultaneously deconvolved. This procedure combines the total $\mathrm{S} / \mathrm{N}$ of all the frames obtained at different periods to determine the light distribution of the extended sources (galaxies), as well as the positions of the point sources (QSOs), since these parameters do not vary with time. The intensities of the point sources, however, are allowed to vary from one image to the other, hence producing the light curves. This technique is particularly well suited to the analysis of $\mathrm{B} 1600+434$, because of the light contamination of the faint $\mathrm{B}$ component by the spiral 


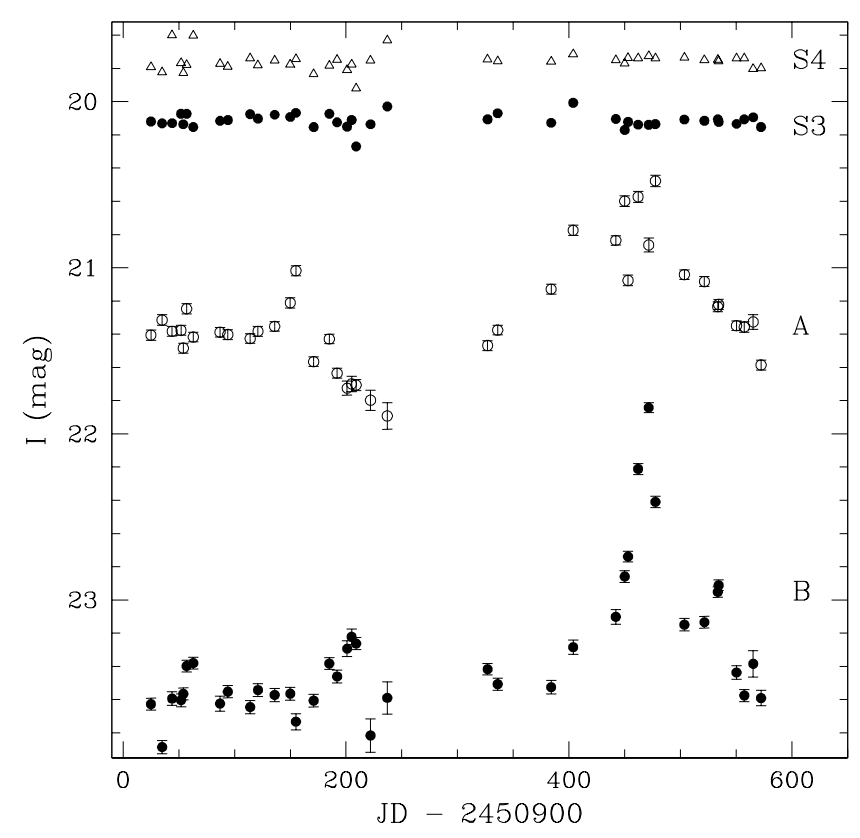

Fig. 2. $-I$-band light curves for B1600 +434 (A and B components) and two reference stars in the field. The plotted value for B is $I(\mathrm{mag})+1.5$, and for S4 I (mag) + 0.4. The magnitudes are calculated relative to calibrated stars in the field. The error bars represent photon noise and PSF errors measured as described in the text (§3). See the electronic edition of the Journal for a color version of this figure.

lensing galaxy. Simultaneous deconvolution of all the frames allows us to derive a high-S/N numerical galaxy model (see Fig. 1). The derived galaxy is in good agreement with the images of B1600+434 obtained with the Hubble Space Telescope (HST; Maller et al. 2000).

Maps of the residuals for each frame are used to check deconvolution results. These maps represent the $\chi^{2}$ fit in each pixel; i.e., the match between the deconvolved model image (reconvolved with the PSF) and the data. By inspecting these residuals, we conclude that the photometry of both components was mainly limited by photon noise.

In order to check for errors introduced by the PSF, we deconvolve two fainter stars in the field that are not used in the construction of the PSF. Light curves for two of these stars, labeled S3 and S4, are displayed in Figure 2. Assuming that these stars are not variable, we use the standard deviation around the mean value as a measure of the photometric accuracy, giving $1 \sigma$ errors of 0.026 and 0.039 mag for S3 and S4, respectively. For each point, we subtract the photon noise in quadrature and attribute the residual error to the PSF. For the QSO images, these estimated PSF errors are added in quadrature to the photon noise in order to model the total errors in the photometry.

\section{LIGHT CURVES}

The $I$-band light curves (Fig. 2) contain 41 data points for each component. As predicted by lens theories, these light curves show that $\mathrm{A}$ is the leading component. $\mathrm{A}$ feature in $\mathrm{A}$ observed at JD 2451050 (1998 September) is repeated in B about 50 days later. In 1999 June ( JD 2451300) there is an increase in the flux from both components. This increase is particularly strong in the B component and represents more than $1 \mathrm{mag}$ in less than 100 days. After the intensity increase, both A and B display several sharp short-term variations. Unfortunately, our light curves are not sufficiently well sampled during this period to recover the exact variations. This suggests that there are variations on timescales faster than the sampling frequency.

The features in the light curves are sufficiently distinct that a rough eyeball estimate directly yields an approximate time delay of about 50 days. Although 41 brightness measurements are a fairly small amount of data, application of standard techniques to measure time delays yields fairly robust results. We analyzed the light curves with the four methods described below.

\section{TIME DELAY MEASUREMENTS}

\subsection{The SOLA Method}

The method of subtractive, optimally localized averages (SOLA) was originally developed for solving inverse problems in helioseismology (Pijpers \& Thompson 1994). The basic idea of this method is to construct an optimal solution, taking into account measurement errors, of any linear inverse problem by taking linear combinations of the measured data. After having been applied successfully in helioseismology, the SOLA method has found application in image reconstruction (Pijpers 1999), in the reverberation mapping of active galactic nuclei (Pijpers \& Wanders 1994), and in the determination of time delays between lensed QSOs (Pijpers 1997).

In the case of lensed quasar images, a transfer function that is a Dirac delta function is positioned at the time delay. With this method, both the delay and the relative magnification of a pair of images are determined. Applied to $\mathrm{B} 1600+434$, a time delay of 55 days and a flux ratio of 0.79 is found. Also determined is a relative offset, e.g., due to a contribution of the lensing galaxy or a foreground object that has not been subtracted, and a (linear) drift between the images, which could occur due to microlensing events with long timescales. Problems can occur if there are higher order relative drifts, such as short-timescale microlensing events that cannot easily be accounted for within this method, which just uses determinations of low-order moments of the transfer function.

The errors are obtained by assuming that the photometric errors are uncorrelated and follow a Gaussian distribution. These propagated errors give a conservative error estimate of \pm 10 days on the time delay value, i.e., $\Delta t=55 \pm 10$ days.

\subsection{The Minimum Dispersion Method}

For the combined data set generated from the A light curve and the shifted B light curve, we estimate the dispersion of the scatter around the unknown mean curve. The true time delay between the images should be manifested as a minimum in the dispersion spectrum (see Pelt et al. 1996 for definitions and notation). Both the simplest stringlength-type statistic, $D_{2}^{2}$, and the smoothed dispersion spectrum, $D_{3}^{2}$ (smoothing parameter $\delta=10$ days), give a clear global minimum at $\Delta t \approx 48$ days.

The precision of this time delay value is estimated using a bootstrap procedure. We first construct the combined light curve from A and the time-delay-shifted B curve $(\Delta t=48)$. This yields a reference curve by median smoothing. From the reference curve, we build bootstrap samples by adding randomized errors. For each sample (altogether 1000) we compute $D_{3}^{2}$ dispersion spectra and find corresponding dispersion minima. The scatter of the dispersion minima for the different bootstrap runs is significant (see Fig. 3), with a formal $1 \sigma$ error estimate of \pm 16 days. 


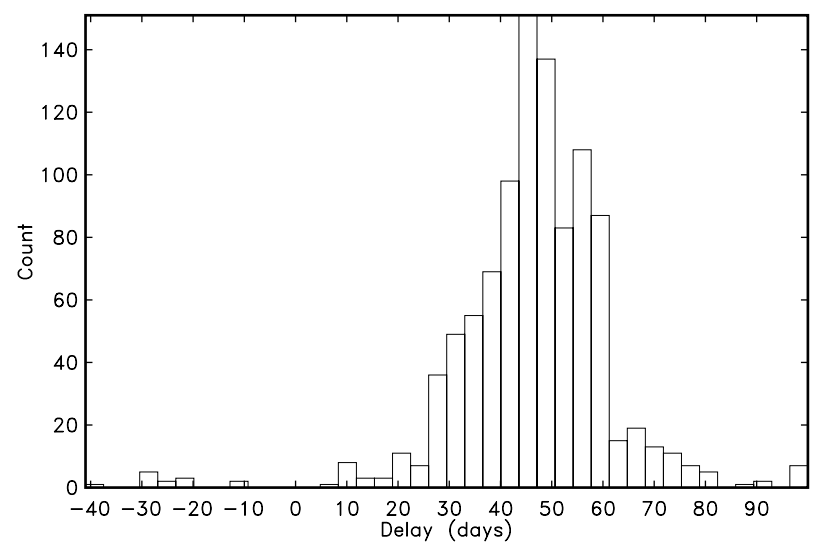

FIG. 3.-Distribution of the time delays for 1000 bootstrap runs with the minimum dispersion method (see $\S 5.2$ ).

\subsection{Model Fit Method}

We model the data with an arbitrary continuous light curve with a fixed and constant sampling. For a given time delay value $\Delta t$, we use $\chi^{2}$ minimization to compare the model curve to the A curve and the appropriately timedelay-shifted and magnitude-offset $\mathrm{B}$ curve. In addition, a linear term in one of the curves can be included to model long-term microlensing effects such as observed in QSO $0957+561$ (Schild \& Smith 1991). The minimization procedure is repeated for a range of time delay values (0 to 100), and the $\chi^{2}$ minimum is obtained with $\Delta t=49$ days and $\Delta m=0.66 \mathrm{mag}$ (i.e., a flux ratio of 0.54 ). An additional small positive linear term for the A component further improves the $\chi^{2}$ value.

A bootstrap method is used to estimate the errors. Two sets of 1000 curves are constructed with the same number of data points as in the data. One set is constructed with the same time sampling as that of our measured data, and the other with a random time sampling. Running the program on these simulated curves results in a $1 \sigma$ standard deviation of 2 days on the set of curves with the same sampling as our measured data, and of 7 days on the curves with random sampling. We could interpret this as an error of 2 days internal to the method, since the sampling stayed the same for each simulated data set, and an error of 7 days on the measured time delay independently of the sampling of the data, but this needs to be investigated further.

\subsection{Iterative Modeling}

Assuming that the additional time-dependent offsets between the time-delay-shifted curves are caused by microlensing, a fourth method based on iterative correction of the flux ratios is applied to the data. We split the light curves into several parts (bins). In one case we chose three bins with lengths determined according to the apparent lengths of the offsets in a certain directions (positive or negative compared to the other curve). In three other cases we separate the curves into 2, 3, and 4 bins with an equal number of data points. For a range of time delay values (40-65 days), we first fit a model (using the $\chi^{2}$ model fit method described above) independently to the separate bins. For each bin and time delay value, an additional constant or linear offset is determined to improve the fit. Finally, new time delay values are determined on these modified curves. The time delays determined in this way turn out to show very little sensitivity to the input values used, or to the different splitting of the curves. The results converge toward a mean value of 51 days with a standard deviation $(1 \sigma)$ of 2 days and magnitude offsets varying from 0.6 to $0.87 \mathrm{mag}$ in the different bins.

\subsection{Results}

The four time delay estimates obtained from the different methods are consistent with one another (see Table 1). An average of the results gives a time delay estimate of 51 days and a flux ratio $\mathrm{A} / \mathrm{B}=1.50$. The flux ratio at radio wavelengths has been measured to be 1.21 (Koopmans et al. 2000). If we assume that the radio flux is not affected by reddening, the B-component is reddened by $0.22 \mathrm{mag}$, corresponding to a factor of 1.23 . At a redshift of 0.41 , this corresponds to $A_{V} \sim 0.25 \mathrm{mag}$, essentially independent of the assumed reddening law.

Except from the iterative modeling, the methods described above yield substantial errors in the time delay. However, the good agreement between the results in addition to the observed time-dependent magnitude offsets in the time-delay-shifted curves (Fig. 4) suggest that the large errors are mostly due to the presence of external variations, e.g., microlensing effects, rather than to the method used to

TABLE 1

Estimated Time Delays and $I$-Band Magnitude DIFFERENCES FOR B1600 + 434 CALCULATED WITH THE Four Methods Described In the TeXt (§ 4)

\begin{tabular}{|c|c|c|}
\hline Method & $\begin{array}{c}\Delta t \\
(\text { days) }\end{array}$ & $\begin{array}{c}\Delta m \\
(\mathrm{mag})\end{array}$ \\
\hline ................. & $55 \pm 10$ & $0.72 \pm 0.007$ \\
\hline Minimum dispersion ...... & $48 \pm 16$ & \\
\hline$\chi^{2}$ fit ........................ & $49 \pm 7$ & $0.66 \pm 0.01$ \\
\hline Iterative fit ................. & $51 \pm 4$ & $0 . \overline{6}-0.87$ \\
\hline
\end{tabular}

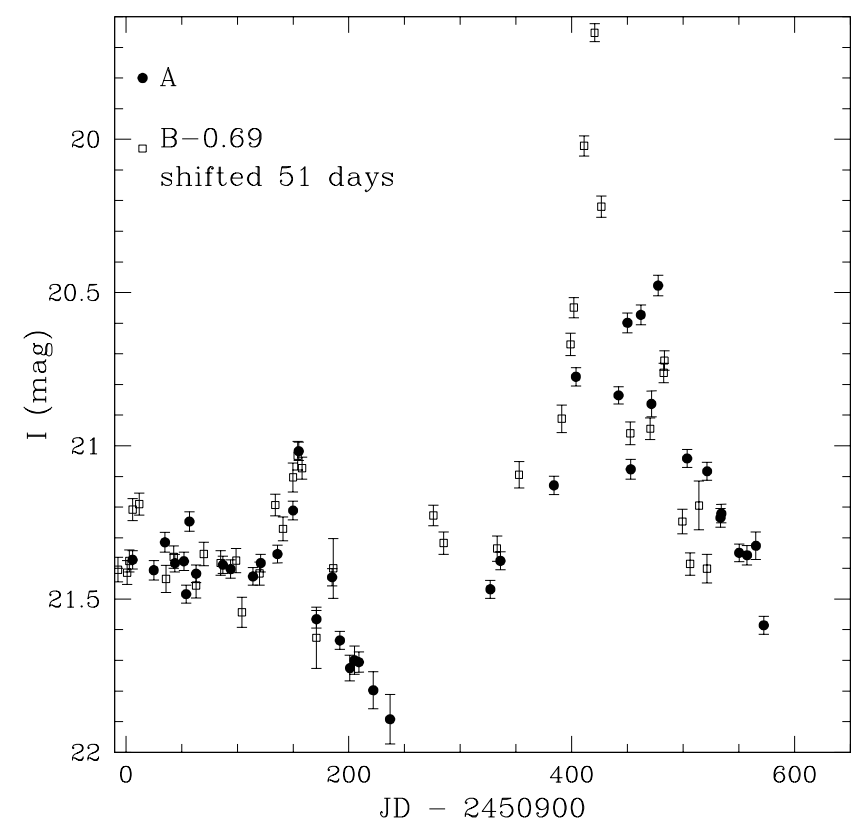

FIG. 4. - Combined light curve from both components of B1600+434. The curve from the B component is shifted forward in time by 51 days and scaled with $-0.69 \mathrm{mag}$. The magnitudes are calculated relative to calibrated stars in the field. The error bars represent photon noise and PSF errors measured as described in the text $(\S 3)$. See the electronic edition of the Journal for a color version of this figure. 
determine the time delay. SOLA and the model fit method correct for slow, linear variations, but not higher order effects. Let us consider a case in which the brighter of the two lensed images undergoes many microlensing events during the time of monitoring. Such short-term variations would be indistinguishable from measurement noise for these methods. They may fail to converge or produce a result that is biased at a level of the order of the errors. With the iterative method, however, we also correct for higher order effects. The small errors from the iterative method compared to the other three methods indicate the presence of such higher order external variations in the light curves.

If no assumptions are made regarding the short-term offsets between the time-delay-shifted curves, we must use the conservative error estimate of \pm 10 days $(1 \sigma)$ on the time delay value. However, if we assume that these external variations are higher order effects, we can use the $1 \sigma$ error estimate of \pm 2 days as found from the iterative method.

\section{6. $H_{0}$ ESTIMATE}

Assuming that microlensing effects affect our $I$-band light curves, a time delay of $51 \pm 4$ days $(95 \%$ confidence level) has been estimated. Applying the galaxy models from Maller et al. (2000) and assuming a $\Omega=0.3$ and $\Lambda=0$ universe, our measured time delay is consistent with a Hubble parameter of $H_{0}=52_{-8}^{+14} \mathrm{~km} \mathrm{~s}^{-1} \mathrm{Mpc}^{-1}(95 \%$ confidence level), where both errors on the model and the time delay are included.

In the model of B1600+434, Maller et al. (2000) assume a constant $M / L$ for the disk and the bulge, and a dark halo that has the same center and the same orientation as the disk. In addition, they include the mass associated with the companion galaxy (see Fig. 1) modeled as an isothermal sphere. The dark matter is modeled as a standard pseudoisothermal elliptical mass distribution (PIEMD; Kassiola \& Kovner 1993), and the exponential profiles of the disk and the bulge are modeled by a two-point PIEMD, also called the chameleon profile (Keeton \& Kochanek 1998; Hjorth \& Kneib 1999). Two types of solutions are found with these models: one with a large dark matter halo core radius, and the other with a nearly singular dark halo. Applying only the solutions with a large dark halo core radius gives $H_{0}=$ $54_{-5}^{+6} \mathrm{~km} \mathrm{~s}^{-1} \mathrm{Mpc}^{-1}$.

For comparison, we also estimate $H_{0}$ using a simple analytical generalized isothermal galaxy model, as described by Witt, Mao, \& Keeton (2000). This method depends only on the time delay and the observed images positions. Using our measured time delay and the image positions from Maller et al. (2000) measured on the $H S T$ image, we obtain $H_{0}=$ $45_{-5}^{+6} \mathrm{~km} \mathrm{~s}^{-1} \mathrm{Mpc}^{-1}(\Omega=0.3$ and $\Lambda=0)$, where errors come only from the time delay and the image positions. This is only an indicative estimate of $H_{0}$ using a very simplified model. Possible shear from ellipticity in the lens galaxy or a mass contribution from the companion galaxy is not taken into account.

Finally, as pointed out by Romanowsky \& Kochanek (1999) and Witt et al. (2000), the time delay depends on the density profile of the lens galaxy. Hence, if the real density profile of the spiral galaxy is different from our models, the errors in $H_{0}$ will increase.

\section{DISCUSSION}

The main goal of our optical monitoring program at the NOT is to measure time delays for as many lensed QSOs as possible, in order to better constrain the mass distribution in lens galaxies. A robust time delay value of $\Delta t=51 \pm 10$ days $(1 \sigma)$ and a flux ratio of 0.69 have been measured from $I$-band light curves of our first target, B1600+434. Assuming that our light curves are affected by external variations, the time delay value is constrained to $51 \pm 4$ days $(95 \%$ confidence level). This value is consistent with a Hubble parameter $H_{0}=52_{-8}^{+14} \mathrm{~km} \mathrm{~s}^{-1} \mathrm{Mpc}^{-1}(\Omega=0.3$, $\Lambda=0$ ), using the models of Maller et al. (2000). We recall that possible systematic errors due to uncertainties in the density profiles and the degeneracy between the relative contribution of disk, bulge, and halo to the mass in the spiral galaxy may increase the uncertainties in the estimated $H_{0}$.

As is evident from Figure 4, and as measured by the various methods to determine the time delay, there are slow additional time-dependent magnitude offsets between the curves. These variations are $\sim 0.2 \mathrm{mag}$ on timescales of a few months. Since the QSO images of B1600+434 pass through the lens galaxy with high optical depths for microlensing, the offsets between the two time-delay-shifted curves (Fig. 4) are likely to be due to microlensing of one of the components. This interpretation is supported by the detection of microlensing in light curves obtained at radio wavelengths (Koopmans et al. 2000), and implies that a significant fraction of the mass in the dark halo consists of massive compact objects. Simulations of microlensing light curves must be carried out in order to determine the lens masses and source sizes that could reproduce the observed variations in our optical light curves (see Wambsganss \& Paczynski 1991; Schmidt \& Wambsganss 1998). Such a microlensing analysis will be published in a separate paper.

Microlensing could also be partly responsible for the sharp event at $\sim$ JD 2451350 in the light curve of the B component. Such high-magnification and short-duration events may occur in cases of microlensing by stars in random motion, whereas the slow variations are typical of microlensing events by stars with velocities following the bulk motion of the galaxy (e.g., Wambsganss \& Kundic 1995). We note, however, that much of this event must be due to intrinsic variations in the QSO, since a significant peak is detected for both components.

Although the temporal sampling of our curves does not allow us to fully disentangle high-frequency microlensing and intrinsic variations, it is sufficient to follow lower order variations, yielding robust time delay estimates fairly independently of the statistical method used. The time delay estimates measured with the four different methods are in agreement with each other. Furthermore, the time delay value of $47_{-9}^{+12}$ days recently estimated from radio measurements (Koopmans et al. 2000) is consistent with our optical measurement. There are thus two independent data sets, one at optical wavelengths ( $I$ band) and one at radio wavelengths, and hence affected in very different ways by microlensing, yielding the same time delay value within the measurement errors.

The remarkably strong variations in this system makes it interesting to study in more detail. The observed microlensing effects may provide important constraints on the MACHO masses in the lens galaxy. Moreover, once $H_{0}$ is known independently from other lenses or other methods, an accurate time delay can be used to constrain the mass distribution between the halo and the bulge of the spiral lens galaxy. In particular, a well-determined time delay and 
flux ratio can contribute to determining the maximum allowed disk mass in the lens galaxy.

We thank the NOT director Vilppu Piirola for granting us observing time for this project on a flexible basis. We are especially grateful to the dozens of visiting observers at NOT who have contributed to this project by performing the scheduled observations. This project was conceived in 1997 while J. H., A. O. J., and J. P. were visiting scientists at the Center for Advanced Study in Oslo. We thank Rolf
Stabell and Sjur Refsdal for inviting us there and for their kind hospitality. We also acknowledge stimulating conversations with Frederic Courbin and the useful comments from the referee. The project was supported in part by the Danish Natural Science Research Council (SNF). I. B. was supported in part by contract ARC 94/99-178 “Action de Recherche Concertée de la Communauté Française (Belgium)" and Pôle d'Attraction Interuniversitaire P4/05 (SSTC, Belgium).

\section{REFERENCES}

Fassnacht, C. D., \& Cohen, J. G. 1998, AJ, 115, 377

Hjorth, J., Burud, I., Jaunsen, A. O., Andersen, M. I., Korhonen, H. Clasen, J. W., \& Østensen, R. 2000, in ASP Conf. Ser., Gravitational Lensing: Recent Progress and Future Goals, ed. T. Brainerd \& C. Kochanek (San Francisco: ASP), in press

Hjorth, J., \& Kneib, J.-P. 1999, ApJ, submitted

Jackson, N., et al. 1995, MNRAS, 274, L25

Jaunsen, A. O., \& Hjorth, J. 1997, A\&A, 317, L39

Kassiola, A., \& Kovner, I. 1993, ApJ, 417, 450

Keeton, C. R., \& Kochanek, C. S. 1998, ApJ, 495, 157

Koopmans, L. V. E., de Bruyn, A. G., Xanthopoulos, E., \& Fassnacht, C. D. 2000 , A\&A, 356, 391

Kundić, T., et al. 1997, ApJ, 482, 75

Magain, P., Courbin, F., \& Sohy, S. 1998, ApJ, 494, 472

Maller, A. H., Simard, L., Guhathakurta, P., Hjorth, J., Jaunsen, A. O., Flores, R. A., \& Primack, J. R. 2000, ApJ, 533, 194

Pelt, J., Kayser, R., Refsdal, S., \& Schramm, T. 1996, A\&A, 305, 97
Pijpers, F. P. 1997, MNRAS, 289, 933 1999, MNRAS, 307, 659

Pijpers, F. P., \& Thompson, M. J. 1994, A\&A, 281, 231

Pijpers, F. P., \& Wanders, I. 1994, MNRAS, 271, 183

Refsdal, S. 1964, MNRAS, 128, 295

Romanowsky, A. J., \& Kochanek, C. S. 1999, ApJ, 516, 18

Schechter, P. L., et al. 1997, ApJ, 475, L85

Schild, R. E. 1990, AJ, 100, 1771

Schild, R. E., \& Smith, R. C. 1991, AJ, 101, 813

Schild, R. E. \& Thomson, D. J. 1995, AJ, 109, 1970 1997, AJ, 113, 130

Schmidt, R., \& Wambsganss, J. 1998, A\&A, 335, 379

Wambsganss, J., \& Kundić, T. 1995, ApJ, 450, 19

Wambsganss, J., \& Paczynski, B. 1991, AJ, 102, 864

Witt, H. J., Mao, S., \& Keeton, C. R. 2000, ApJ, 544, in press (preprint astro-ph/0004069) 